\title{
Immunology in China: the past, present and future
}

\begin{abstract}
Xuetao Cao
Over the past decade immunological research in China has developed rapidly. This commentary describes the history, summarizes the present research funding system, institutions and representative work, and discusses the future of immunological research in China.
\end{abstract}

\begin{abstract}
$\triangle \mathrm{s}$ an important frontier discipline of the life Asciences and medicine, immunology contributes greatly to the understanding of health and disease and is helpful in the diagnosis, treatment and prevention of human disease. As Chinese civilization is one of the oldest in the world, China has a long history of immunological research, with Chinese immunologists having made notable achievements in several areas of this field.
\end{abstract}

\section{The past}

Edward Jenner (1749-1823) is often credited as being the founder of immunology for generating a vaccine against smallpox. In 1798 , Jenner inoculated a boy using pus from the arm of a milkmaid suffering from cowpox, and then noted that the boy did not develop disease when inoculated with smallpox. Vaccination as a method for inducing immunity to infectious disease soon became widely accepted and ultimately led to the eradication of smallpox. However, the idea of immunity may have existed long before Jenner in traditional Chinese medicine.

Documents show that as early as AD 1000, the ancient Chinese custom existed of having children inhale powders made from the crusty skin lesions of patients recovering from smallpox ${ }^{1,2}$. Even earlier, around AD 303, Ge Hong, a famous doctor in China, had written "kill the dog that bites, and use its

Xuetao Cao is with the Institute of Immunology and National Key Laboratory of Medical Immunology, Second Military Medical University, Shanghai 200433, China.

e-mail: caoxt@public3.sta.net.cn brain to prevent recurrence of rabies". In AD 649, Sun Simiao, another famous Chinese doctor, said that the most effective method of preventing a recurrence of rabies was to "cover the people with the sick dog's brain" (Fig. 1). It therefore seems that for more than a millennium, doctors in China have been attempting to prevent or even cure infectious diseases by prophylactic immunization.

Modern immunology is based on experimental observations that may be 'translated' to the clinic to prevent and treat diseases. In China, modern immunological research dates back to the 1930s. At that time, two prominent figures at Peking Union Medical College (founded in 1917 with the support of the Rockefeller Foundation) were doing important research. Sizhi Liu (19041983) purified and quantified antibodies, whereas Shaowen Xie (1903-1995) successfully cultured and expanded rickettsia in chicken embryos, leading to the immunological detection of rickettsia and an inactivated vaccine for the prevention of rickettsiosis. Two other prominent figures, both from Shanghai, were $\mathrm{He} \mathrm{Yu}$ (1903-1988), who first proposed the hypothesis of 'allergy-mediated pathogenesis of rheumatic fever' in 1933, and Feiqing Lin

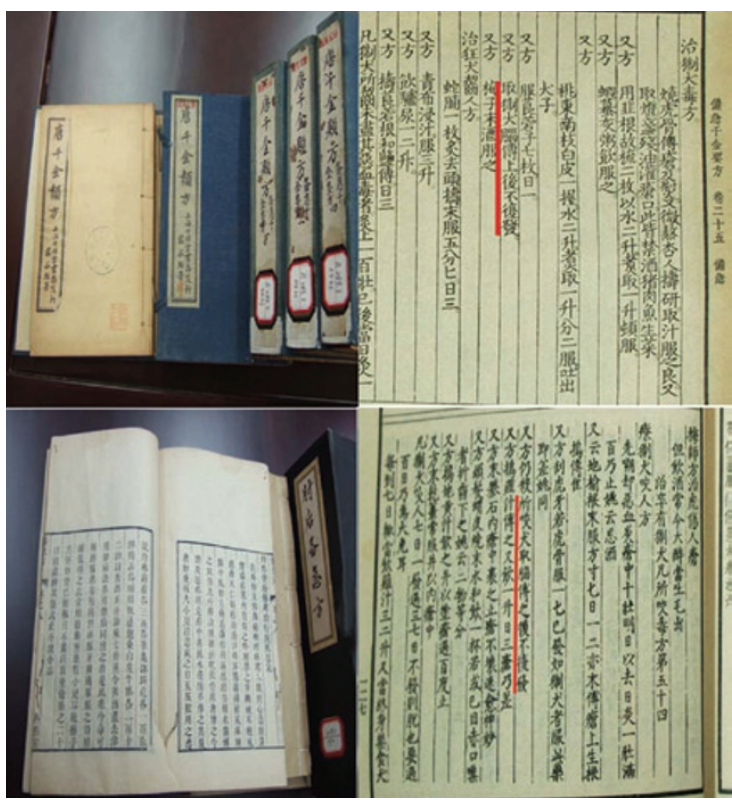

Figure 1 The idea of 'prophylactic immunization' for preventing the recurrence of rabies is presented in ancient books of Chinese traditional medicine. Top left, Bei Ji Qian Jin Yao Fang by Sun Simiao (581-682), published circa AD 649 , and the related description of preventing the recurrence of rabies by "covering the people with the sick dog's brain" (red line; top right). Bottom left, Zhou Hou Bei Ji Fan by Ge Hong (283-363), published circa AD 303, and the related description of preventing the recurrence of rabies by "killing the dog that bites, and using its brain for the people who will be safe without relapse of rabies" (red line; bottom right).

(1904-1998), who contributed to the understanding of the immune response against bacterial infection ${ }^{3}$. In the 1960s, Fangzhou $\mathrm{Gu}$, former president of the Chinese Academy of Medical Sciences, developed an industrial system for producing vaccines against 
infectious diseases. In the 1970s, Yunde Hou identified a new type of interferon- $\alpha$ and 10 years later successfully put a recombinant protein on the market for the treatment of chronic hepatitis B.

After the Cultural Revolution in China (1966-1976), the government made great efforts to develop science and technology in China by funding new projects for scientific research, encouraging young scholars to go abroad, and promoting international scientific exchanges and collaborations. Since then, many immunologists, including Guizhen Yang, Wufei Zheng and Zhenzhou Long, have helped translate immunology textbooks into Chinese and have organized advanced courses in modern immunology. Tianxing Ye (1915-1999) of the Second Military Medical University was the first in China to edit the textbook Theory and Practice of Immunology, published in March 1979. In the 1980s, many Chinese immunologists who had spent time in cutting-edge laboratories in the United Kingdom, the United States, Australia and Japan returned to China. These distinguished immunologists made outstanding contributions to the development of immunological research in China. For example, Denian Ba conducted the first clinical trial of adoptive cellular therapy for cancer patients in 1986; Weifeng Chen established an experimental system for basic research in T cell development; and Beifeng Shen prepared and modified monoclonal antibodies to leukemic cells and diverse pathogens.

The Chinese Society for Immunology was founded in 1988. It now has over 2,500 members, with seven academic committees in different fields, six official periodicals in Chinese, and one official journal in English (Cellular \& Molecular Immunology). For the past 20 years, more and more young immunologists, many educated in the United States and Europe, have established laboratories, departments or institutes of immunology in China's universities and have published in international peer-reviewed journals since the late 1990s (ref. 4).

\section{Research funding and institutions}

The Chinese economy has grown an average of $9.4 \%$ every year for the past 26 years. To promote the development of science and technology, the Chinese government now spends $1.23 \%$ of its gross domestic product on funding for basic and applied scientific research. Along with material sciences and information sciences, biomedical research has been listed as one of the three most important and internationally competitive fields to be preferentially supported by the government. In the past 10 years, funding in the field of life sciences and medicine, including basic and applied immunological research, has increased considerably. Immunologists in China, like scientists in other fields, may obtain funding by several routes. Most funding comes from the Ministry of Science and Technology of China (MOST) and the National Natural Science Foundation of China (NSFC). MOST prefers to support large target-oriented projects, aiming to strengthen fundamental research and 'hi-tech' development in several national

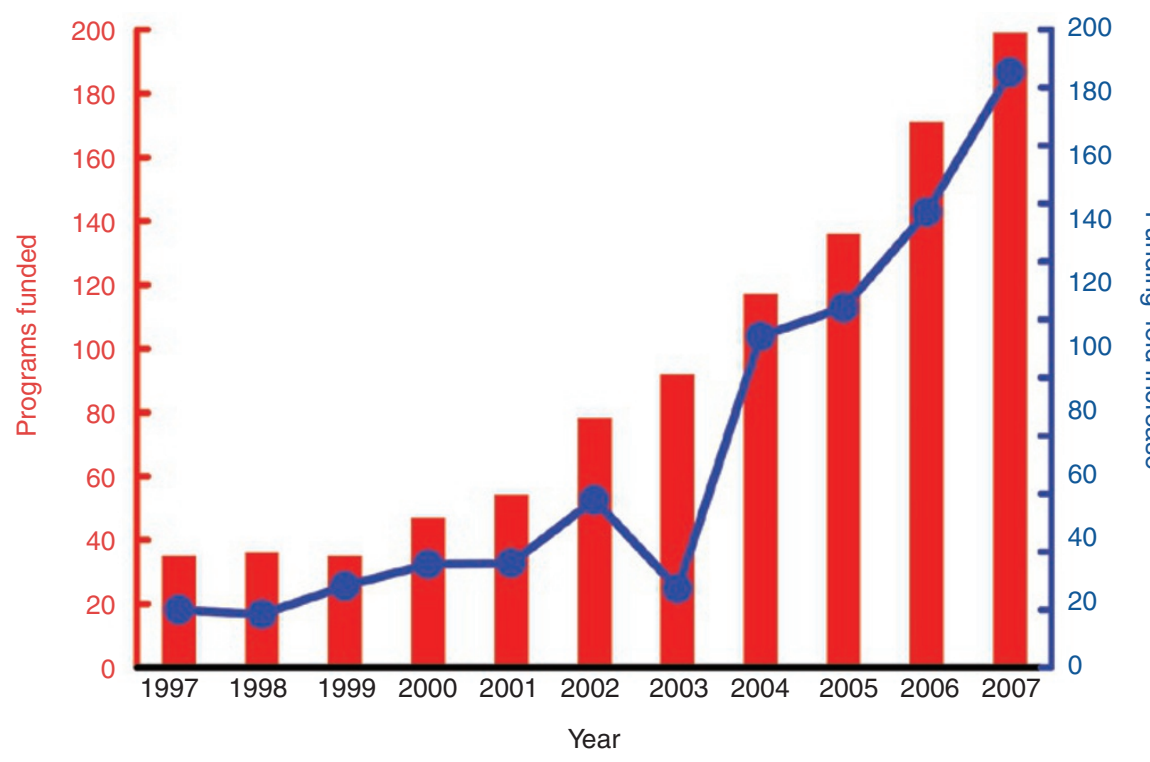

Figure 2 NSFC funding for immunological research in China, 1997-2007. Red bars indicate the number of programs funded by the immunology division of the NSFC in the field of immunology; the blue curve indicates the 'fold increase' in NSFC funding for immunological research in China.

programs, including the National Basic Research Program of China (973 Program) and the National High-Tech R\&D Program (863 Program). In the past 10 years, Chinese immunologists have received financial support from MOST to establish research teams and bases to undertake basic immunological research and to develop immunological products such as recombinant cytokines, humanized antibodies and vaccines.

The NSFC usually supports and encourages individual immunologists to focus on single key scientific issues. Thus, basic research in cellular and molecular immunology has been supported that covers almost all the fields of immunology, including the development, phenotypic characteristics and function of immune cells and their subsets; recognition and signaling in innate immunity; the activation and regulation of adaptive immunity; the identification and structural studies of immune molecules; the cellular and molecular mechanisms of immune regulation and tolerance; and the generation of immunological memory. Innovation and application of new immunological techniques is encouraged. The integration of immunology with genomics, proteomics, systems biology and imaging techniques is selectively supported, which allows systemic and quantitative investigation of immune responses. In particular, this holistic approach allows immunologists to study interactions between various immune cells in the immune network. NSFC has launched 'Key Program' projects to support leading groups to explore major scientific and technological issues in immunology. For example, one Key Program (1999-2003) investigated the immune regulation and organ transplantation, and another Key Program (2004-2008) is for the investigation of the structural basis of immune recognition. In general, funding for each program has increased 1.5-fold since 1997. Overall, funding from NSFC for immunological research has increased about 10-fold in the past 10 years (Fig. 2). In addition, immunologists in China can also obtain funding from the Ministry of Education, the Ministry of Public Health and local governments.

However, the number of world-class institutions and immunology laboratories is still limited in China. Most immunological laboratories are in medical schools and universities. As the biggest scientific organization in China, the Chinese Academy of Science (CAS) has begun to establish centers and laboratories for immunological research, such as the Center for Infection and Immunity at the Institute of Biophysics in Beijing, and the Institut Pasteur of Shanghai. To promote 
translational research, MOST has set up a series of National Centers and National Key Laboratories in universities and other institutions. In 2006, MOST set up the National Key Laboratory for Medical Immunology at the Second Military Medical University in Shanghai, with an emphasis on both basic and applied immunological research to diagnose, treat and prevent diseases.

\section{Present research}

Pursuing the tradition of investigating immunological mechanisms in the pathogenesis of conditions such as cancer, infection, organ transplantation, allergy and autoimmune diseases, Chinese immunologists continue to take advantage of clinical resources, including a huge population of patients and a bank of clinical samples of various diseases, to define the mechanisms of pathogenesis and to elucidate new immunological approaches for the diagnosis, treatment and prevention of disease. There are active research groups in the areas of hematopoiesis and the development of immune cells, autoimmunity and tolerance, molecular and structural immunology, infection and immunity, transplantation immunology, tumor immunology, immunotherapy and vaccine development, and the design and application of immunological techniques.

Because chronic infection with hepatitis $B$ virus (HBV) is one of the most common health problems in China, Chinese immunologists are making substantial efforts to investigate the immunological mechanisms of chronic infection with HBV and other aspects of liver immunology. Yumei Wen (Fudan University, Shanghai) is well recognized for contributions to the analysis of HBV replication in liver tissues of patients infected with HBV and for the design and clinical application of yeast-derived complexes of hepatitis B surface antigen and hepatitis B immunoglobulin as a therapeutic vaccine for patients chronically infected with $\mathrm{HBV}^{5}$. Bo Tien (CAS, Beijing) has identified an HBV-specific peptide bound to heat-shock protein gp96 derived from the liver tissues of patients infected with HBV and has proposed the use of this HBV-specific peptide as a vaccine for chronic infection with $\mathrm{HBV}$ and for HBV-related hepatocellular carcinoma ${ }^{6}$. Yuzhang Wu (PLA Institute of Immunology, Chongqing) has developed a therapeutic vaccine for HBV infection (mimovirus), which is formed through electrostatic interactions by the self-assembly of a cationic peptide containing 18 lysine residues, a cytotoxic T lymphocyte-epitope peptide of hepatitis B surface antigen amino acids 28-39, and a plasmid encoding interleukin 12. This vaccine induces cytotoxic $\mathrm{T}$ lymphocyte responses specific for hepatitis B surface antigen amino acids $28-39$ (ref. 7). Zhigang Tian (University of Science and Technology of China, Hefei) is an expert in natural killer (NK) cell immunobiology and has done a series of experiments evaluating regulation of NK cell function in the liver. Tian's group focuses on the regulatory innate immune network of NK, NKT and Kupffer cells in hepatitis and has found that activation of Toll-like receptor 3 (TLR3) on Kupffer cells prevents lipopolysaccharide-induced acute hepatitis in mice by decreasing TLR4 expression and that activation of TLR3 on NK cells decreases the severity of NKT cell-mediated liver injury ${ }^{8}$.

Several groups are investigating the development and functional regulation of T cells. Weifeng Chen (Peking University) is heading a group studying $\mathrm{T}$ cell development in the microenvironment of the thymus. By analyzing the maturation program of $\mathrm{CD}^{+}$singlepositive thymocytes in Aire-deficient mice, they have shown that the development of $\mathrm{CD}^{+}{ }^{+}$single-positive cells in the medulla is critically dependent on a functionally intact medullary epithelial cell compartment ${ }^{9}$. Gang Pei (Shanghai Institute for Biological Sciences at CAS) has found that the adaptor protein $\beta$-arrestin- 1 is required for the maintenance of CD4 T cell survival and that high expression of $\beta$-arrestin- 1 causes autoimmunity, which links this molecule to the pathogenesis of multiple sclerosis ${ }^{10}$. Heading a group neighboring Pei's laboratory, Bin Sun has been investigating the development of $\mathrm{T}$ helper type $1\left(\mathrm{~T}_{\mathrm{H}} 1\right)$ and $\mathrm{T}_{\mathrm{H}} 2$ cells and the pathogenic function of $\mathrm{T}_{\mathrm{H}} 1$ cells in experimental autoimmune uveitis. Inspired by the wide use of pertussis toxin as an adjuvant to induce $\mathrm{T}_{\mathrm{H}} 1$-mediated organ-specific autoimmune disease, this group has shown that pertussis toxin can activate dendritic cells (DCs) to release more interleukin 12 through the Erk kinase pathway and that pertussis toxin-primed DC 'preferentially' drive $\mathrm{T}_{\mathrm{H}} 1$ responses $^{11}$.

The molecular and cellular mechanisms of immune regulation always attract much attention because dysregulation of immune function is involved in many diverse diseases. A key issue in this field is how to balance the immune response and immune (negative) regulation. 'Balancing' the human body to prevent disease is an important philosophical tenet of traditional Chinese medicine, in the theory of yin (negative regulation) and yang (positive regulation). Similarly, in the immune system there are feedback regulators or controllers that initiate and activate immune responses (Fig. 3). Thus, Chinese immunologists are interested in the functions of regulatory T cells, regulatory DCs and regulatory macrophages (or alternatively activated macrophages) in controlling immune responses, which benefit the host by eliminating invading pathogens while not causing damage to host tissues.

Given that mature DCs, having recognized and taken up pathogens, migrate to secondary lymph organs and present the processed anti- 
gens to T cells and initiate T cell immunity, my group has investigated the effect of the stromal microenvironment on the fate of mature DCs. Unexpectedly, we have found that splenic stroma can drive mature DCs to continue proliferating and further differentiate into a DC subset that inhibits $\mathrm{T}$ cell responses, thus challenging the dogma that mature DCs are terminally differentiated and die after interactions with $\mathrm{T}$ cells. Our results demonstrate the importance of the immune organ microenvironment in the determination of immune cell function, outline a previously unknown mode of negative regulation of the $\mathrm{T}$ cell response at level of the antigen-presenting cell, and demonstrate how DCs switch from initiators with $\mathrm{T}$ cell-activating capacity to negative regulators with suppressive function for T cell responses ${ }^{12}$. Jingwu Zhang's group has extended their previous work describing $\mathrm{T}$ cell vaccination of irradiated autologous $\mathrm{T}$ cells from patients with multiple sclerosis. This group has shown that induction of Foxp $3^{+} \mathrm{CD} 4^{+}$regulatory $\mathrm{T}$ cells is integral to the therapeutic effect of $\mathrm{T}$ cell vaccination and, notably, that the $\mathrm{T}_{\mathrm{H}} 1$ cytokine interferon- $\gamma$ is involved in the induction of such cells ${ }^{13}$.

After recognizing microbial conserved pathogen-associated molecule patterns, pathogen-recognition receptors such as TLRs transduce signals to activate innate immune cells to produce proinflammatory cytokines and type I interferon and then contribute to the activation of the adaptive immune responses. The group of Hongbin Shu (Peking University) has identified the protein VISA ('virus-induced signaling adaptor'; also called MAVS, IPS-1 or Cardif) as a critical component of the virus-triggered interferon- $\beta$ signaling pathway ${ }^{16}$. Although full activation of the responses of pathogen-recognition receptors is necessary for the elimination of invading pathogens, unbalanced production of proinflammatory cytokines and type I interferons in immune responses may lead to immunopathology. To address this issue, Chinese immunologists have demonstrated that $\beta$-arrestin-1, the phosphatases SHP-2 and SHIP-1, and PECAM-1 (CD31) negatively regulate TLR signaling-triggered production of proinflammatory cytokines in macrophages ${ }^{15,17}$, which adds new insight into the balanced control of innate immune responses. Notably, one group headed jointly by Yangxin Fu and Hong Tang (CAS, Beijing) has shown that resting T cells, including $\mathrm{CD}^{+}{ }^{+} \mathrm{CD} 25^{-} \mathrm{Foxp}^{-}$and $\mathrm{CD} 8^{+} \mathrm{T}$ cells, have an unexpected function in tempering the virus- or TLR-triggered production of proinflammatory cytokines, which identifies a previously mode of regulating the innate response by the adaptive immune system ${ }^{14}$.

Three groups, headed by Zihe Rao, Gorge Gao and Beifeng Shen (Beijing), are active in the important field of structural immunology. Also, groups at the R\&D Centers in Shanghai and Beijing, supported by international pharmaceutical companies, are using genomic and proteomic approaches to identify the genes and molecules involved in the immune response and tolerance, with the hope of identifying new targets for the treatment of disease.

Several approaches for cancer immunotherapy are also being investigated. Wei He's group (Chinese Academy of Medical Sciences, Beijing) has identified a previously unknown structural basis by which $\gamma \delta \mathrm{T}$ cells recognize tumor cells through the CDR3 $\delta$ region $^{18}$. They are now expanding $\gamma \delta \mathrm{T}$ cell populations in vitro for future clinical trials of adoptive immunotherapy for ovarian cancer. A phase II clinical trial of DC-based chemo-immunotherapy for patients with advanced colon cancer with liver metastasis has been approved by the Chinese Food and Drug Administration. In addition, several therapeutic antibodies have been approved for the market, including licartin ( ${ }^{131}$ I-labeled monoclonal antibody HAb18G-CD147), from Zhinan Chen's group, now being used to treat patients with advanced hepatocellular carcinoma ${ }^{19}$.

\section{The future}

With increased funding support from the central government, the 'national team' for immunological research in China is expanding rapidly. In recent years, more and more young scientists, with well recognized experience and notable achievements in immunological scientific research, have returned to China from the United States, Europe and Japan and are now actively involved in the promotion of immunological research in China. The Chinese Society for Immunology is playing an increasingly important role in bringing Chinese immunologists together to promote scientific communications in the national network and to share their achievements and challenges in immunological research by organizing annual meetings and advanced courses in immunology. Many Chinese colleagues who went abroad years ago are now becoming leading immunologists, for example, Yong-Jun Liu and Lieping Chen, and are active in promoting international collaboration between China and other countries. Every year many top international immunologists are invited to come to China to give seminars about new ideas and new advances in immunology, which helps bring Chinese immunologists to the forefront of modern immunology. All such activities provide a firm basis for the further development of immunological research in China in the future.
With the support of two rounds of the 973 National Program in Immunology (2002-2006 and 2007-2011), a national network for immunological research in China has been established, and summit meetings are organized twice yearly on specific 'hot topics'. The chief aim of the present program (2007-2011) is to investigate mechanisms of immune recognition and immune regulation, with six subtopics covering the structural basis of antigen recognition, innate immune recognition and its regulation, the immunobiology of DCs, NK cells and T cell subsets, and the pathogenesis of autoimmune disease and allergy. Immunologists in this program are being encouraged to collaborate with scientists with different disciplinary backgrounds, to integrate projects in genomics, proteomics, systems biology and imaging science, to undertake basic research into key questions in immunology, and to use biological-pharmaceutical platforms for translational research or for the application of immune techniques to clinical settings. With such joint efforts, immunological research in China should soon be at the forefront of the international stage.

\section{Concluding remarks}

Following the rapid development of the Chinese economy, immunological research is being supported with more funding from the government, and more immunological laboratories have been established in the past decade. The present funding system not only supports new, innovative and interdisciplinary basic research focused on better understanding of the immune system but also translational or applied research of immunological techniques. As a result, Chinese immunologists have become more active in the international community and are making efforts to advance the science of immunology for the benefit of everyone.

1. Langer, W.L. Sci. Am. 234, 112-117 (1976).

2. Abbas, A.K., Lichtman, A.H. \& Pillai, S. Cellular and Molecular Immunology $6^{\text {th }}$ edn. (Saunders Elsevier, Philadelphia, 2007).

3. Ba, D. Chin. J. Immunol. 20, 7-9 (2004).

4. Cao, X. et al. J. Immunol. 161, 6238-6244 (1998).

5. Yao, X. et al. Vaccine 25, 1771-1779 (2007)

6. Meng, S.D., Gao, T., Gao, G.F. \& Tien, P. Lancet 357 528-597 (2001).

7. Wu, Y.Z. et al. J. Virol. 76, 10264-10269 (2002).

8. Jiang, W. et al. Proc. Natl. Acad. Sci. USA 102 17077-17082 (2005).

9. Li, J. et al. Proc. Natl. Acad. Sci. USA 104, 18175 18180 (2007).

10. Shi, Y. et al. Nat. Immunol. 8, 817-824 (2007).

11. Hou, W. et al. J. Immunol. 170, 1728-1736 (2003).

12. Zhang, M. et al. Nat. Immunol. 5, 1124-1133 (2004).

13. Wang, Z. et al. J. Clin. Invest. 116, 2434-2441 (2006).

14. Xu, L.G. et al. Mol. Cell 19, 27-40 (2005).

15. Wang, Y. et al. Nat. Immunol. 7, 139-147 (2006).

16. An, H. et al. Immunity 25, 919-928 (2006).

17. Kim, K.D. et al. Nat. Med. 13, 1248-1252 (2007)

18. Xu, C. et al. Mol. Immunol. 44, 302-310 (2007).

19. Xu, J. et al. Hepatology 45, 269-276 (2007). 